\title{
Development Of An DDF Unit For Power Communication
}

Lin Bing-hua

Sanming Power Supply Bureau of Fujian Electric Power Co.1td,Sanming 365000,Fujian,China

\begin{abstract}
DDF unit is an importantpart in power communication field. It is the connection and connection equipment between digital multiplex equipment, digital multiplex equipment and program-controlled switching equipment or data service equipment and other professional equipment. However, the traditional DDF unit needs to be tested by $2 \mathrm{M}$ error code, which is inconvenient to carry and will not be used if it is not for the communication staff. Especially in the remote substation, substation operation and maintenance personnel at the scene of the failure, but can not match. This paper provides a DDF communication unit, to overcome the shortcomings of existing technology, substation operation and maintenance personnel can visually see the connectivity, distance to deal with the fault, shorten the fault time, improve work efficiency.
\end{abstract}

Key words: DDF unit; Power communication; detection device

DDF (Digital Distribution Frame) unit is an important part in the field of electric power communication。 It is suitable for the transmission rate is $2 \mathrm{Mb} / \mathrm{s} \sim 155 \mathrm{Mb} / \mathrm{s}$ digital terminal equipment or SPC digital signal wiring and switching circuit, switching and scheduling, with test function, convenient maintenance, flexible circuit line of business change and adjustment. Therefore, the equipment has a wide range of application, and it is the necessary transmission equipment for the digital transmission room and the program-controlled switchboard room. The position of the digital distribution frame is shown in figure 1 .

\section{Background Technology}

DDF (Digital Distribution Frame) unit, also called high-frequency distribution frame, has more and more advantages in digital communications. The DDF unit can make the digital stream digital communication equipment connected as a whole, is between the wiring, digital multiplex equipment of digital multiplex equipment and program-controlled switching equipment or non business connections between devices with equipment, wiring, jumper, switching and testing function flexible.

The DDF unit consists of several pairs of $2 \mathrm{M}$ terminals, carrying the important business such as relay protection and dispatching automation. There are two reasons for business failure, one is terminal equipment failure, the other is channel fault. The traditional detection method is $2 \mathrm{M}$ error code, the disadvantage is that the instrument must be carried anytime, and if it is not communication staff will not be used. Especially in remote sites, substation operators at the scene, but noway to match.

\section{DDF Unit Structure Of Power Communication}

The aim of the invention is to overcome the shortcomings of existing technology, provides a new DDF unit, substation operation and maintenance personnel can visually see the connectivity, distance to deal with the fault, shorten the fault time, improve work 
efficiency.

The utility model relates to an DDF unit, and the structure of the utility model is characterized in that the utility model comprises metal plate body, inscription strip, connecting terminal and LED lamp.

The appearance of the DDF unit is gray, can be turned, and the rotation angle is 180 degrees to facilitate the operation of the connector plug. A DDF unit is a unit board 20 system, divided into two rows, each row on the 10.

The metal plate is made of full copper material and has good corrosion resistance.

The inscription for labeling, length of the unit plate length corresponding to the outside with a layer of transparent plastic, inside a long strip of paper, up and down each row.

The connecting terminal is an unbalanced coaxial connector with $75 \mathrm{ohm}$, the surface is plated with gold, the contact resistance is low, and the connection is reliable. 20 a unit board of a system with 4 connecting terminals. In general, the connecting terminal 3 is connected with the service side, and the connecting terminal 5 is connected with the service side, and the connecting terminal 4 is connected with the hair of the equipment side, and the connecting terminal 6 is connected with the side of the device.

The LED light is a system of 4 , connectivity LED lamp 7 indicates connection terminals 5 and 6, LED lamps 8 indicates connection terminal connectivity 3 and 4 LED, indicating lamp 9 connection terminals 3 and 5 connectivity, LED connectivity indicator lamp 10 connection terminals 4 and 6 . For lights, no lights.

In normal use, as shown in Figure 3, the vertical plug connection terminals 3 and 4 connected to the circuit in Figure 2, R1, D1, diode D1 lights, LED lights lit 8; connection terminals 5 and 6 connected, R2, D2, diode D2 lights, LED lights, 7 lights. LED lights 9 and 10 are not lit.

When the loopback test is shown, as shown in Figure 4, that is, the horizontal plug is connected with the connecting terminals 3 and 5, the R3 and the D3 work, the diode D3 is bright, and the LED lamp 9 is bright; when the connecting terminals 4 and 6 are connected, the R4 and the D4 work, and the LED lamp 10 is bright. LED lights 7 and 8 are not lit.
In summary, the present invention has the following advantages over prior art:

1.Simple and intuitive, not communication staff, but also through the LED light directly observe the channel, the initial judgment is equipment problems or channel problems, reduce misoperation, misoperation probability.

2. When the communication personnel software loop back test, on-site substation operators can check the ring back through the LED lamp is wrong, remote processing failure.

Brief description of drawings

Figure 2 is a schematic diagram of a panel of the present invention. Label description 1 metal plates; 2 Ming bars; 3, 4, 5, 6 connecting terminals; 7, 8, 9, and 10 LED lights.

Figure 3 is an internal circuit diagram of the present invention.

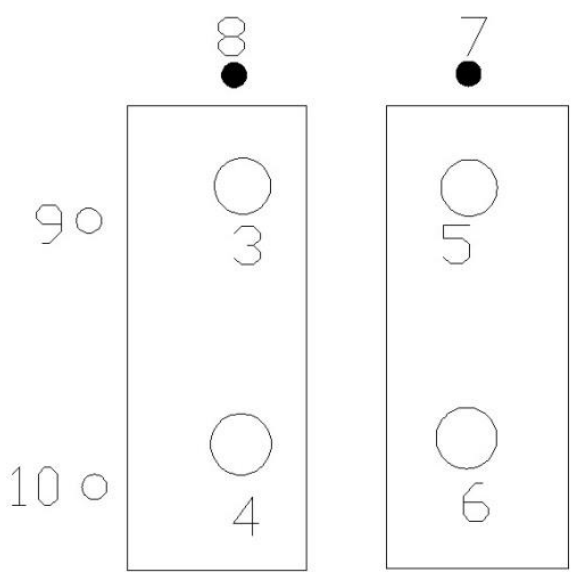

Figure 4 A connection diagram when the connection terminal is used.
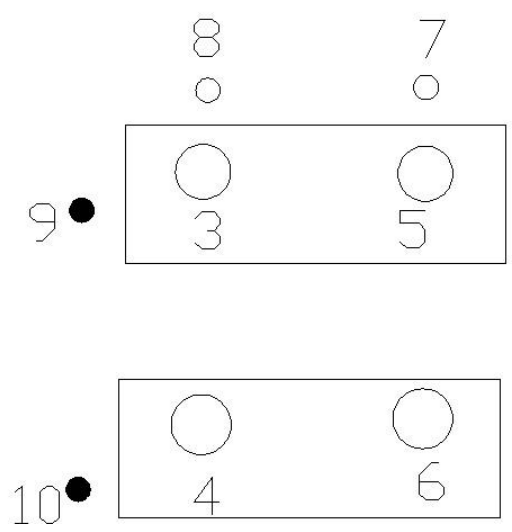

Figure 5 The connection diagram of the connection terminal ring back. 


\section{3 application example analysis}

\section{Embodiment 1}

The utility model relates to an electric communication DDF unit, which comprises a metal plate body, a inscription strip, a connecting terminal and a LED lamp. The appearance of the DDF unit is gray, can be turned, and the rotation angle is 180 degrees to facilitate the operation of the connector plug. A DDF unit is the unit board of the 20 system. It is divided into two rows and 10 pairs on each row. The metal plate is made of full copper material and has good corrosion resistance. The inscription for labeling, length of the unit plate length corresponding to the outside with a layer of transparent plastic, inside a long strip of paper, up and down each row. The connecting terminal is an unbalanced coaxial connector with $75 \mathrm{ohm}$, the surface is plated with gold, the contact resistance is low, and the connection is reliable. 20 a unit board of a system with 4 connecting terminals. In general, the connecting terminal 3 is connected with the service side, and the connecting terminal 5 is connected with the service side, and the connecting terminal 4 is connected with the hair of the equipment side, and the connecting terminal 6 is connected with the side of the device. The LED light is a system of 4 , connectivity LED lamp 7 indicates connection terminals 5 and 6, LED lamps 8 indicates connection terminal connectivity 3 and 4 LED, indicating lamp 9 connection terminals 3 and 5 connectivity, LED connectivity indicator lamp 10 connection terminals 4 and 6. For lights, no lights.

In normal use, as shown in Figure 3, the vertical plug connection terminals 3 and 4 connected to the circuit in
Figure 2, R1, D1, diode D1 lights, LED lights lit 8; connection terminals 5 and 6 connected, R2, D2, diode D2 lights, LED lights, 7 lights. LED lights 9 and 10 are not lit. When the loopback test is shown, as shown in Figure 4, that is, the horizontal plug is connected with the connecting terminals 3 and 5, the R3 and the D3 work, the diode D3 is bright, and the LED lamp 9 is bright; when the connecting terminals 4 and 6 are connected, the R4 and the D4 work, and the LED lamp 10 is bright. LED lights 7 and 8 are not lit. The LED light 7 in Fig. 1 corresponds to the diode D1 in Fig. 2, and the LED light 8 in Fig. 1 corresponds to diode D2 in Fig. 2, and the LED light 9 in Fig. 1 corresponds to diode D3 in Fig. 2, and the LED light 10 in Fig. 1 corresponds to diode D4 in Fig. 2.

\section{Conclusion}

The DDF (Digital Distribution Frame) unit is a wiring and connection equipment between the digital multiplex equipment, the digital multiplex equipment and the program-controlled switching equipment or the data service equipment and other specialized equipment.

The invention can directly observe the channel condition through the LED lamp, and preliminarily judge whether the equipment is a problem or a passage problem, and reduce the probability of misoperation and misoperation, and is simple and direct. At the same time, when the communication personnel software loop back test, on-site substation operators can check the ring back through the LED lights are wrong, remote processing of faults, shorten the failure time, improve work efficiency. 


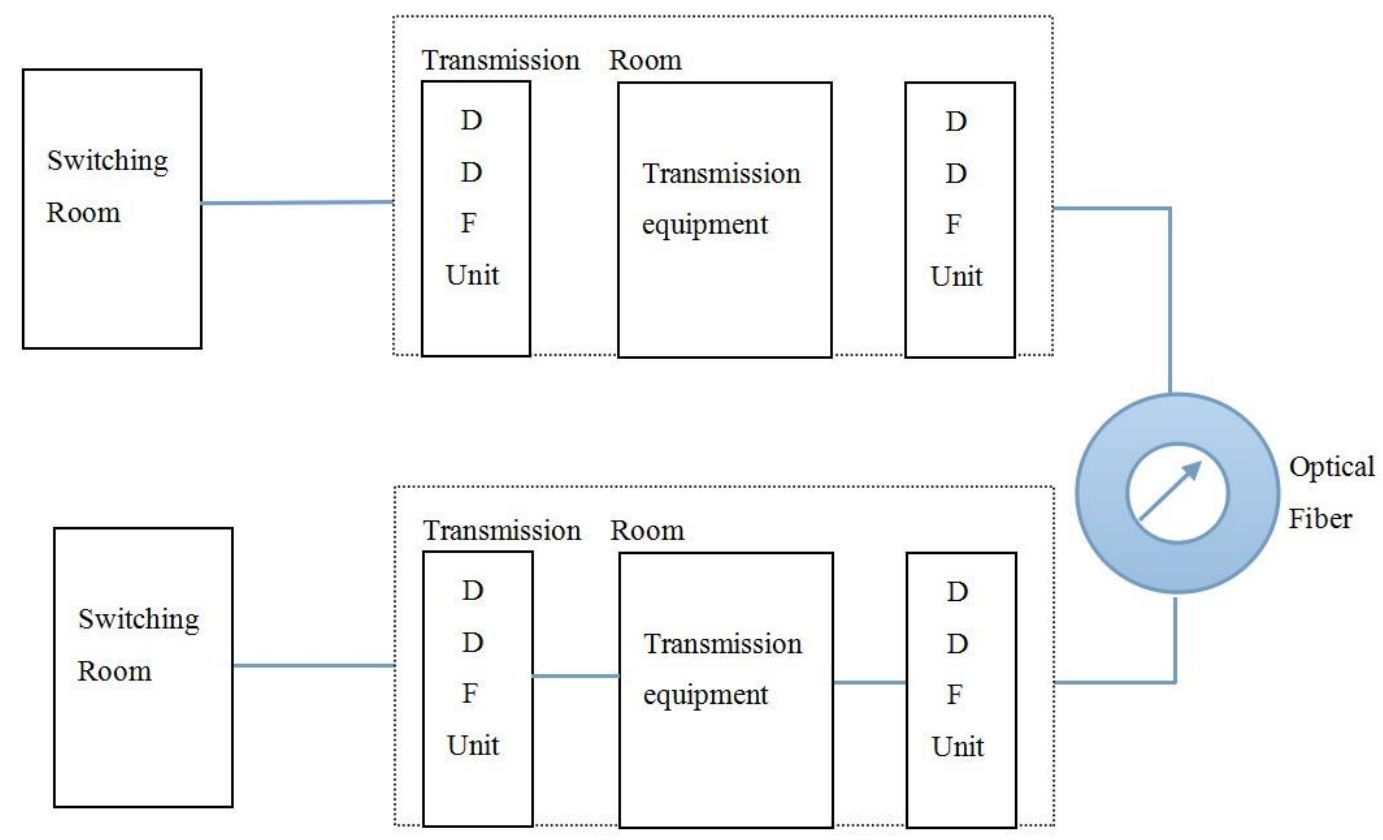

Figure 1 Location diagram of the DDF unit in the transmission

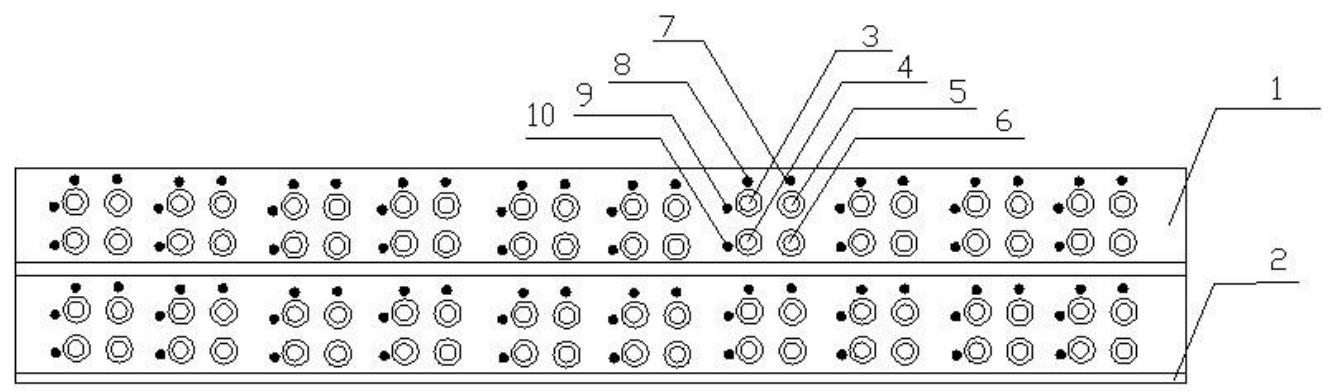

Figure 2 A schematic diagram of a panel of the present invention

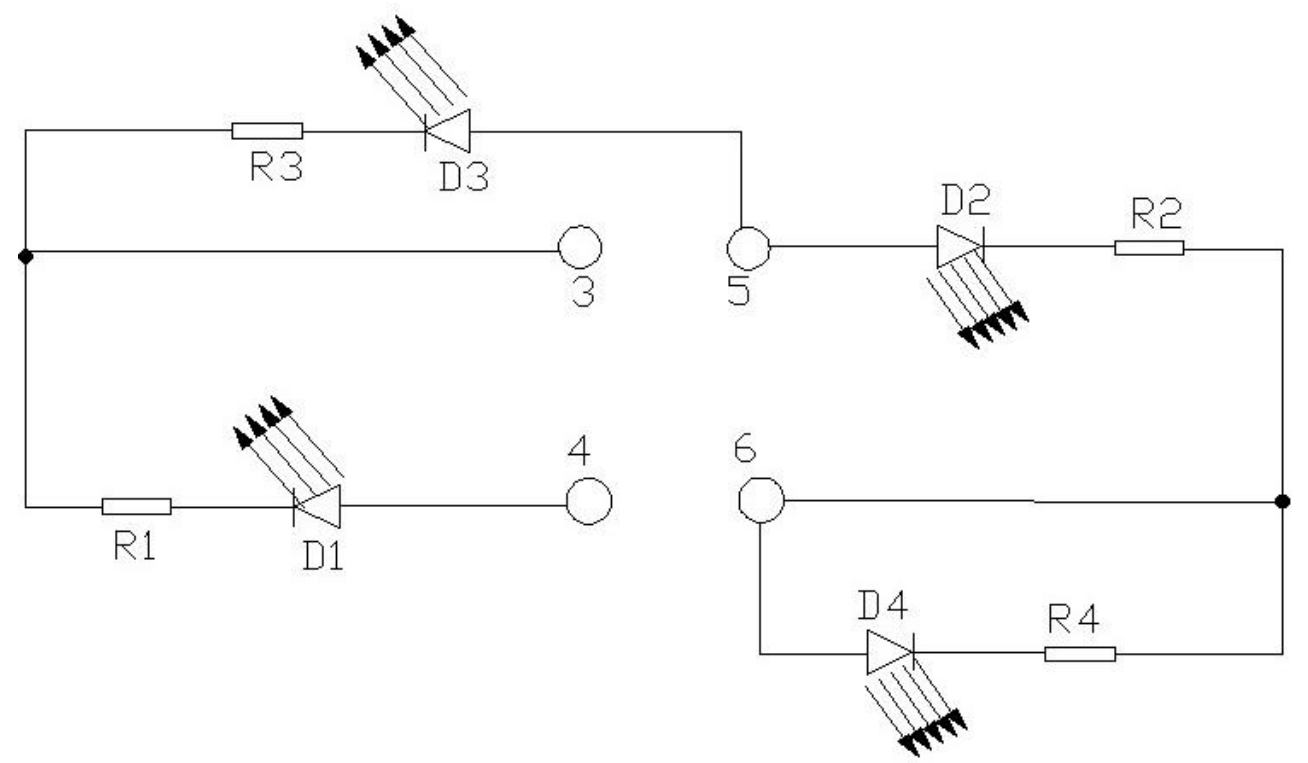

Figure 3 An internal circuit diagram of the present invention 